\title{
Improving uniformity of atmospheric-pressure dielectric barrier discharges using dual frequency excitation
}

\author{
Y Liu ${ }^{1,2}$, F J J Peeters' 1 , S A Starostin ${ }^{3}$, M C M van de Sanden ${ }^{1,2}$ and H W de \\ Vries $^{1}$ \\ ${ }^{1}$ Dutch Institute for Fundamental Energy Research, Eindhoven, 5612 AJ, The \\ Netherlands \\ ${ }^{2}$ Eindhoven University of Technology, Eindhoven, 5612 AZ, The Netherlands \\ ${ }^{3}$ FUJIFILM Manufacturing Europe B.V., Tilburg, 5047 TK, The Netherlands
}

Email: H.W.deVries@differ.nl

\begin{abstract}
This letter reports a novel approach to improve the uniformity of atmospheric-pressure dielectric barrier discharges (DBDs) using a dual-frequency (DF) excitation consisting of a low frequency (LF) at $200 \mathrm{kHz}$ and a radio frequency (RF) at $13.56 \mathrm{MHz}$. It is shown that due to the periodic oscillation of the RF electric field, the electron acceleration and thus the gas ionization is temporally modulated i.e. enhanced and suppressed during each RF cycle. As a result, the discharge development is slowed down with a lower amplitude and a longer duration of the LF discharge current. Hence, the RF electric field facilitates improved stability and uniformity simultaneously allowing a higher input power.
\end{abstract}

Atmospheric-pressure diffuse non-thermal plasma has various applications in industry [1-6]. One of the common ways to create large area non-thermal plasmas at atmospheric pressure (AP) is the dielectric barrier discharge (DBD) $[1,2,4]$. However, AP-DBD is typically filamentary resulting in a strong spatial nonuniformity of the plasma, restricting its use for demanding applications such as deposition of high quality thin films [1,2]. The less common diffuse modes of AP-DBD can expand application fields in plasmaassisted surface engineering and therefore are in the focus of scientific and industrial interest [7-13]. Due to the periodic re-ignition, the breakdown mechanism of DBDs determines the discharge phenomenology. To get a stable homogeneous DBD, during the breakdown the secondary electron emission at the cathode ( $\gamma$ emission), related to the negative surface charge of the dielectric [14,15], the trapped ions in the gas [9] and the long-lived metastables [16], has to be enhanced compared to the ionization in the gas bulk ( $\alpha$ ionization) [17]. During the discharge development, the ionization has to be slow enough to avoid a large electronic avalanche. This can be achieved by enhancing the multiple step ionization process e.g. Penning ionization [18] and by reducing the gas voltage as soon as or before the ionization level becomes high enough to localize the electric field [19]. Besides, even though a diffuse discharge is obtained, it could transit into a filamentary mode when the power dissipated in the discharge increases beyond a certain limit [20]. In this study, we report an approach to improve the plasma uniformity together with a higher input power in an industrially relevant cylindrical electrode geometry by applying an extra $13.56 \mathrm{MHz}$ radio frequency (RF) voltage on the $200 \mathrm{kHz}$ low frequency (LF) voltage. 
A schematic of the atmospheric-pressure roll-to-roll plasma reactor is presented in figure 1 . The discharge was ignited between a flat bottom electrode and a curved top electrode with a radius of $60 \mathrm{~mm}$ and a width of $45 \mathrm{~mm}$. Both the electrodes were covered by $0.1 \mathrm{~mm}$ thick polymeric substrates as the dielectrics. The electrode temperature was maintained at $30^{\circ} \mathrm{C}$ by means of an oil circulation system. The smallest distance between the two electrodes was $0.5 \mathrm{~mm}$. The gas mixture was injected from the left side of the discharge area in figure 1, while the substrates were transported at $40 \mathrm{~mm} / \mathrm{min}$ in the same direction as the gas flow. The flow rate of the gas mixture $\left(\mathrm{Ar} / \mathrm{O}_{2} / \mathrm{N}_{2}\right)$ was controlled at $5 \mathrm{slm} / 1 \mathrm{slm} / 1 \mathrm{slm}$ (standard litre per minute). The DBD was excited by $200 \mathrm{kHz}$ LF (SEREN L3001) and 13.56 MHz RF (SEREN R601) power sources which were superimposed and applied to the top electrode through a home-made matching circuit. The injected power of both power sources was modulated at $625 \mathrm{~Hz}$ with a pulse width of $800 \mu \mathrm{s}$ and a duty cycle of 50\%. The discharge voltage and current were measured by a high voltage probe (Tektronix P6015A) with $75 \mathrm{MHz}$ bandwidth and a current transformer (Pearson model 4100), respectively. The discharge emission from the front view of the discharge area was obtained using a digital camera (Olympus OM-D E-M10 Mark II). An intensified charge-coupled device (ICCD) camera (PI MAX3), triggered by the applied voltage, was employed to collect the discharge emission from the side view of the gas gap with a macro lens (Tamron AF $90 \mathrm{~mm}$ ).

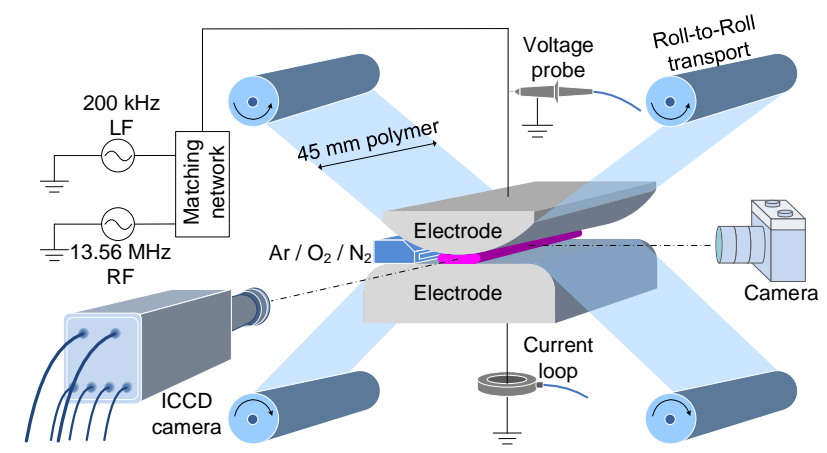

Figure 1. Experimental set-up for the dual-frequency (DF) dielectric barrier discharge together with the electrical and optical diagnostic methods.

Figure 2 shows the voltage and current waveforms within one LF cycle $(5 \mu \mathrm{s})$ recorded for the fixed electrode configuration and gas mixture with increasing amplitude of the RF voltage $\left(U_{\mathrm{RF}}\right)$. In the single $\mathrm{LF}$ discharge $\left(U_{\mathrm{RF}}=0 \mathrm{kV}\right)$, two current peaks can be clearly identified from the measured current, see figure 2(a). In the DF discharges $\left(U_{\mathrm{RF}}>0 \mathrm{kV}\right)$, both the current and the voltage include LF and RF signals. By doing fast Fourier transform (FFT) of the original waveforms, the LF and RF components of both voltage and current can be separated (results not shown here). The amplitude of the LF voltage is relatively constant $(2.2 \pm 0.1 \mathrm{kV})$ in this study. With $U_{\mathrm{RF}}$ increasing $(0 \mathrm{kV}$ to $0.58 \mathrm{kV})$, the LF current increases from $0.12 \mathrm{~A}$ to $0.16 \mathrm{~A}$, while the RF current increases significantly from $0 \mathrm{~A}$ to $0.97 \mathrm{~A}$. This is mainly induced by an increase of the capacitive component of the RF current due to the solid dielectrics and the gas gap [21]. Moreover, by doing FFT filtering, signals above $10 \mathrm{MHz}$ are removed, and the LF component of both voltage and current can be extracted, see figure 2(b)-(d).

Figure 3 shows the time-integrated images of the discharge emission from the side and front-view of the discharge area. From the side-view images, the discharges are relatively uniform with maximal emission intensity close to the electrodes. With $U_{\mathrm{RF}}$ increasing, the discharge area gradually expands, and the intensity of the discharge emission increases. From the front-view images and the corresponding intensity profiles, the single $\mathrm{LF}$ discharge $\left(U_{\mathrm{RF}}=0 \mathrm{kV}\right)$ is non-uniform with numerous filaments appearing in the discharge area. With $U_{\mathrm{RF}}$ increasing, the uniformity of the discharge is gradually improved, while filaments can be hardly observed in figure $3(\mathrm{~d})$. 

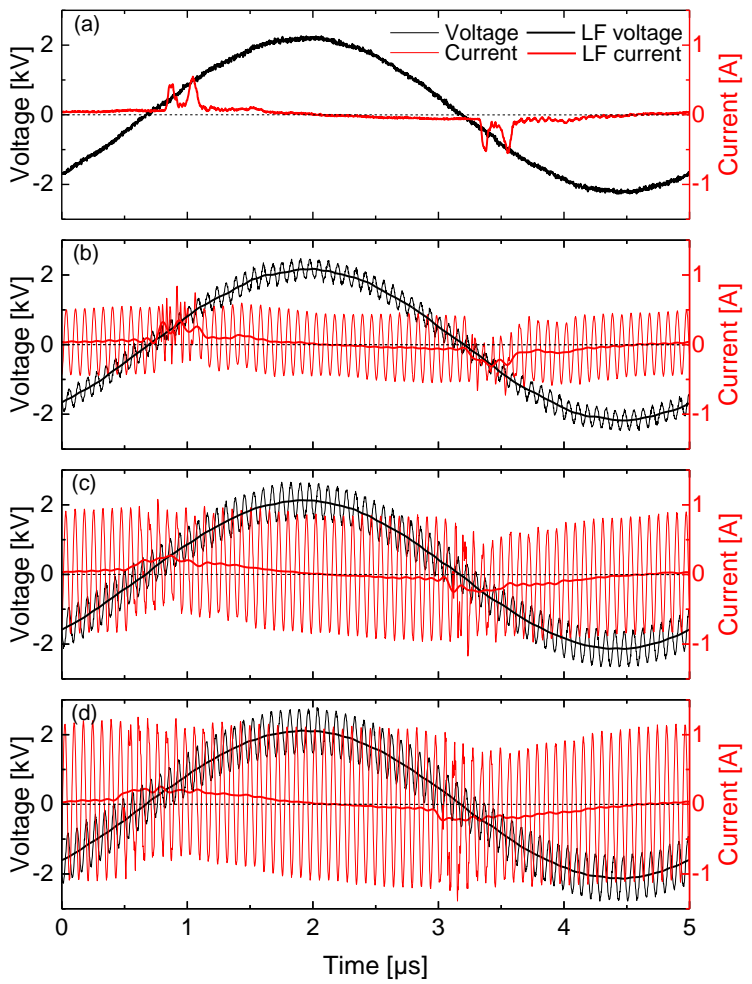

Figure 2. Original and fast Fourier transform (FFT) filtered current-voltage waveforms with RF voltage amplitude of (a) $0 \mathrm{kV}$, (b) $0.26 \mathrm{kV}$, (c) $0.46 \mathrm{kV}$ and (d) $0.58 \mathrm{kV}$.
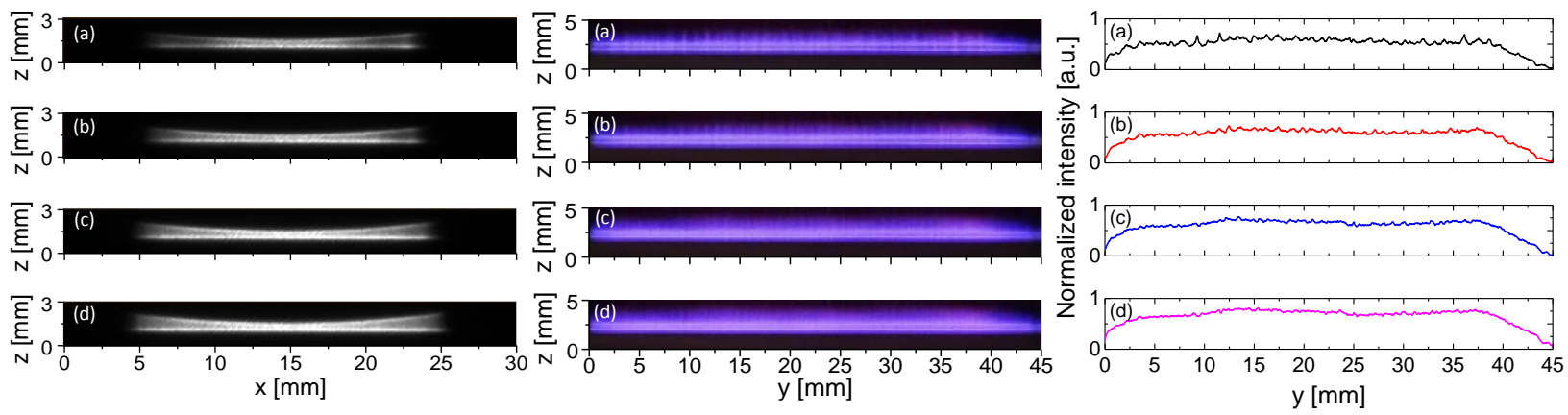

Figure 3. Side-view ICCD images with $1.6 \mathrm{~ms}$ exposure time (left), front-view images with $4 \mathrm{~ms}$ exposure time (middle) and normalized intensity profile of the front-view images (right) with RF voltage amplitude of (a) $0 \mathrm{kV}$, (b) $0.26 \mathrm{kV}$, (c) $0.46 \mathrm{kV}$ and (d) $0.58 \mathrm{kV}$.

By controlling the delay between the camera gate and the voltage, single shot side-view ICCD emission intensities with gate width of $10 \mathrm{~ns}$ was obtained at various phases. The emission profiles in the discharge area were integrated in the horizontal direction ( $x$ direction in the side-view images in figure 3 ), and the phase-resolved discharge emission was obtained by collating and reconstructing the results, as shown in figure 4. The flat grounded electrode is situated at $z=0 \mathrm{~mm}$, and the centre of the curved powered electrode is situated at $z=0.5 \mathrm{~mm}$ where the gap distance is the minimum. The emission intensity is normalized to the maximum in figure 4(d). From figure 4, the LF discharge $\left(U_{\mathrm{RF}}=0 \mathrm{kV}\right)$ is in a transient mode occupying approximately $40 \%$ of the LF cycle $(5 \mu \mathrm{s})$. With the increasing of $U_{\mathrm{RF}}$, both the discharge duration and the momentary emission intensity are enhanced. 

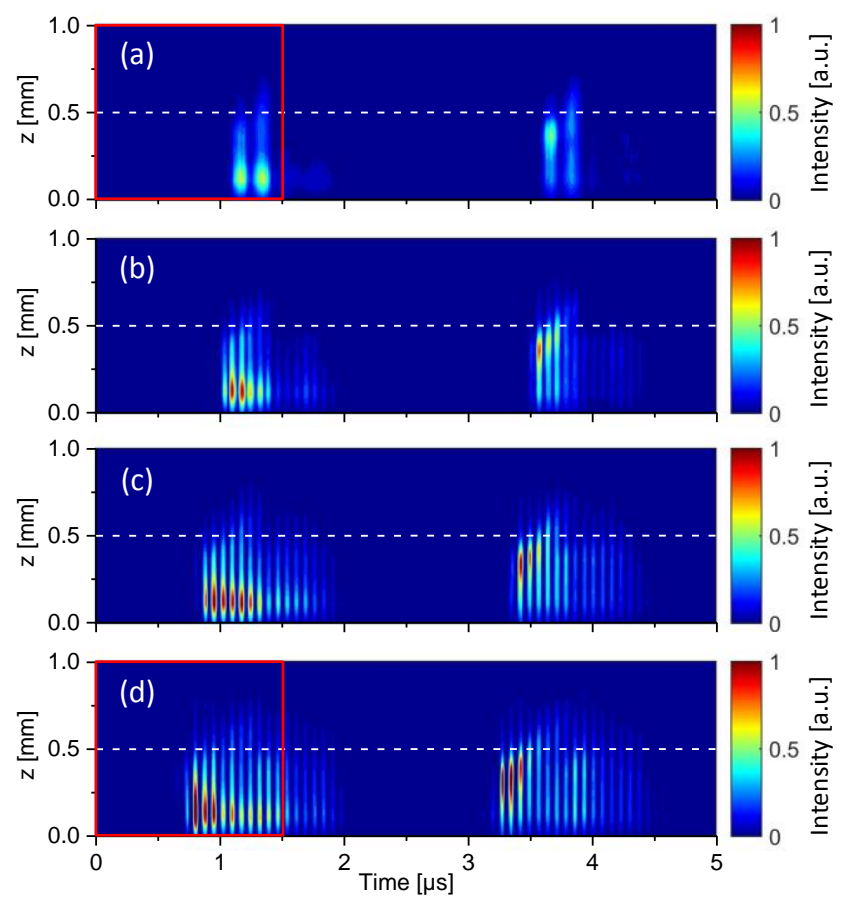

Figure 4. Phase-resolved discharge emission within one LF cycle ( $5 \mu \mathrm{s})$ of the DF discharges with the RF voltage amplitude of (a) $0 \mathrm{kV}$, (b) $0.26 \mathrm{kV}$, (c) $0.46 \mathrm{kV}$ and (d) $0.58 \mathrm{kV}$. The dash lines indicate the position of the minimal gaseous gap $(0.5 \mathrm{~mm})$. The surrounded areas correspond to the detailed emissions in figure 5 (a) and (b).

The detailed phase-resolved emission profiles in the surrounded areas in figure 4 together with the gas voltage and the discharge current are illustrated in figure 5(a) and (b), respectively. The estimated values of the gas breakdown voltage, the maximal amplitude of the LF discharge current, the input power and the normalized discharge emission intensity as a function of $U_{\mathrm{RF}}$ are presented in figure 6 .

From figure 5(a), the discharge emission shows a structure with a high luminescence close to the instantaneous cathode and a bulk region, which under these conditions is identified as a high-current "glowlike" mode and is transited from a low-current "Townsend-like" structure (too weak to see here) [22]. In this case, for the continuously running discharge, the gas breakdown voltage was estimated at about 0.67 $\mathrm{kV}$ which is much lower than the first breakdown $(\sim 1.9 \mathrm{kV}$, result not shown here) in the beginning of each pulse train when the "memory effect" is not established [17]. As the discharge further develops, the positive space charge, initially accumulated in the anode region due to the difference in the mobility of electrons and positive ions, starts to affect the electric field profile. This will lead to the enhancement of the electric field and ionization rate in the cathode direction, resulting in the formation of the fast propagating ionization wave directed from the anode to the cathode [22]. Thus a transition to the glow-like mode occurs, accompanied by a fast jump in the current and the emission intensity by a few orders of magnitude, see figure 5(a).

Similar to the single LF discharge, the DF discharge $\left(U_{\mathrm{RF}}>0 \mathrm{kV}\right)$ is transient within the LF cycle but is also temporally modulated by the RF voltage, see figure $5(\mathrm{~b})$. The ignition starts when the momentary LF voltage is about $0 \mathrm{kV}$, while the total gas voltage $\left(U_{\mathrm{LF}}+U_{\mathrm{RF}}\right)$ reaches about $0.65 \mathrm{kV}$ (similar to the single $\mathrm{LF}$ discharge in figure 5(a)). From figure 6 , with $U_{\mathrm{RF}}$ increasing, the total gas breakdown voltage stays relatively stable with a value of $0.7 \pm 0.05 \mathrm{kV}$. Moreover, a clear transition from a "Townsend-like" structure to a "glow-like" structure is observed in the initial stage of the DF discharge, see figure 5(b). For the DF discharge, due to the difference in mobility, the ions can be regarded as static during the RF period ( $\sim 74$ $\mathrm{ns})$, while the electrons can follow the fast varying RF electric field. The field in the gas gap is enhanced 
during the positive half cycle and suppressed during the negative half cycle of the RF voltage, leading to a temporally synchronous oscillation of the electron acceleration and thus the gas ionization. As a result, the ionization propagation within the LF cycle is slowed down, as confirmed by the clear "Townsend-like" to "glow-like" transition in figure 5(b) as well as the gradually decreasing amplitude of the LF discharge current in figure 6. As previously introduced, with a slower discharge development, a large electronic avalanche and thus a filamentary discharge can be avoided [17]. Hence, the RF modulation can be an effective approach to improve the stability and uniformity of the discharge, as shown in figure 3 . Furthermore, from figure 6, increasing $U_{\mathrm{RF}}$ also allows a higher input power together with a higher discharge emission intensity.

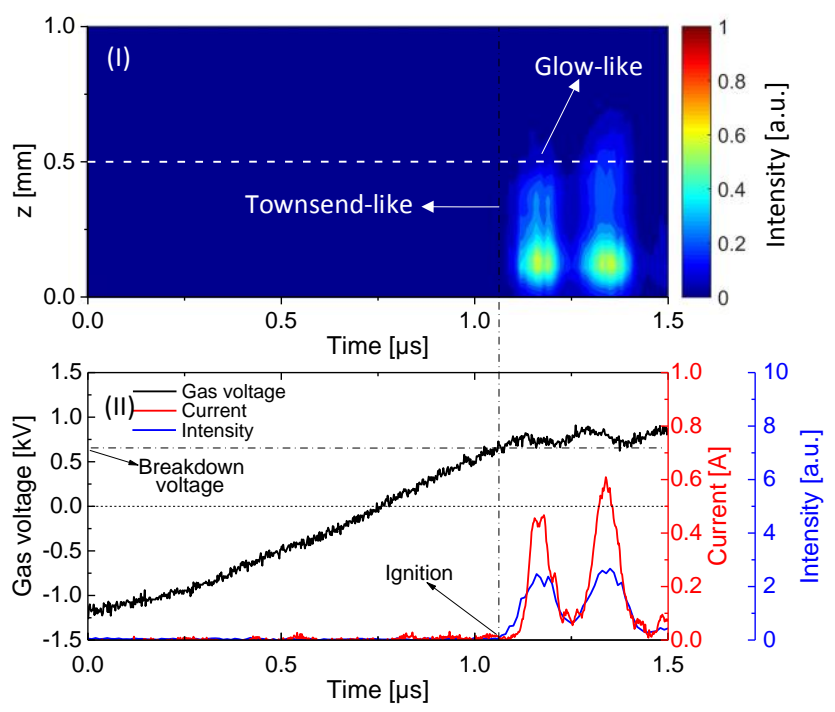

(a)

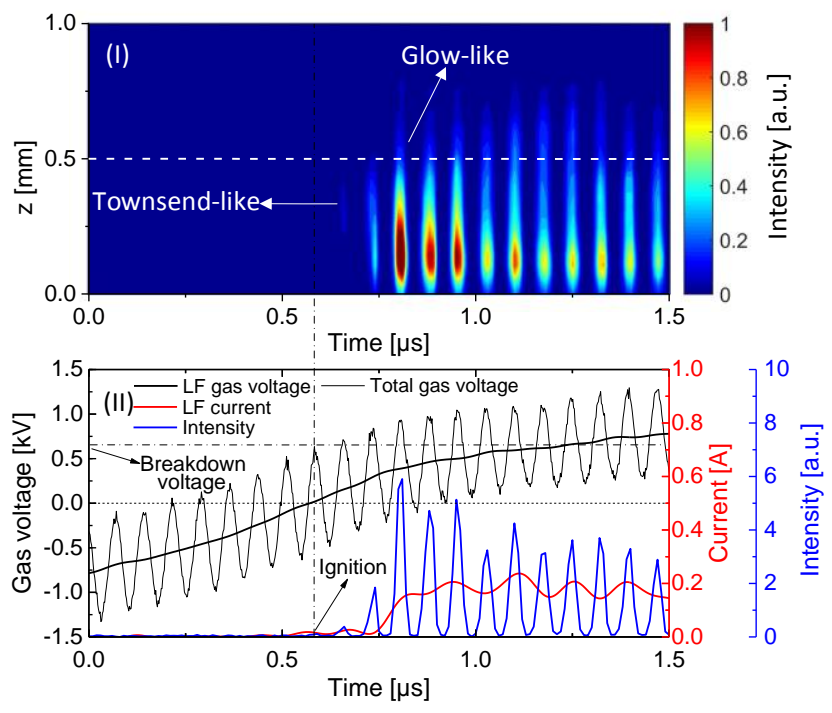

(b)

Figure 5. Phase-resolved discharge emission, gas voltage and discharge current in the initial stage of the discharges with RF voltage amplitude of (a) $0 \mathrm{kV}$ and (b) $0.58 \mathrm{kV}$. 


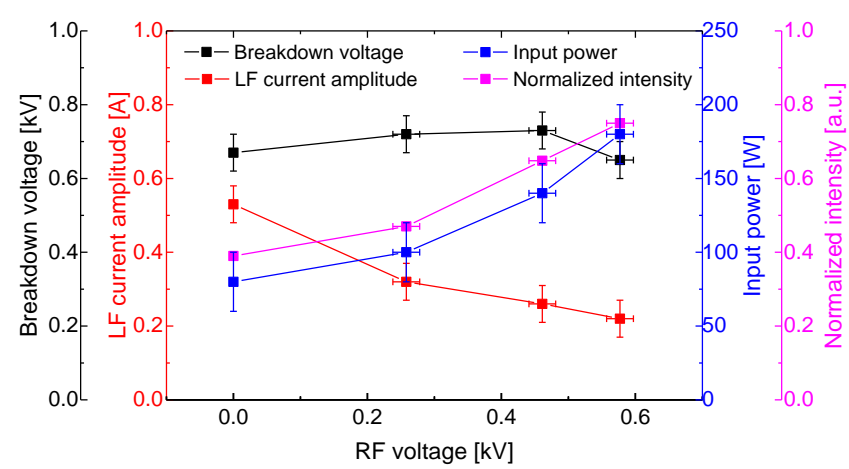

Figure 6. Variations of the breakdown voltage, the maximal LF current amplitude, the input power and the normalized discharge emission intensity as a function of the RF voltage amplitude.

To further understand the DF discharge mechanism, the FFT of the phase-resolved discharge emission in figure $4(\mathrm{~d})$ is studied, see figure 7 . Both fundamental and harmonics of $f_{\mathrm{LF}}, f_{\mathrm{RF}}$ and $f_{\mathrm{RF}} \pm f_{\mathrm{LF}}$ signals can be observed. The FFT amplitudes of the fundamental signals $\left(f_{\mathrm{OLF}}, f_{\mathrm{ORF}}\right.$ and $\left.f_{\mathrm{ORF}} \pm f_{\mathrm{OLF}}\right)$ as a function of the $\mathrm{RF}$ voltage amplitude are shown in figure 8 . With $U_{\mathrm{RF}}$ increasing, both the fundamental components of $\mathrm{LF}$ and $\mathrm{RF}$ are moderately increased. The $f_{\mathrm{ORF}} \pm f_{\mathrm{OLF}}$ components, however, exhibit a more significant increase, indicating a stronger response of the discharge to the combined frequencies.

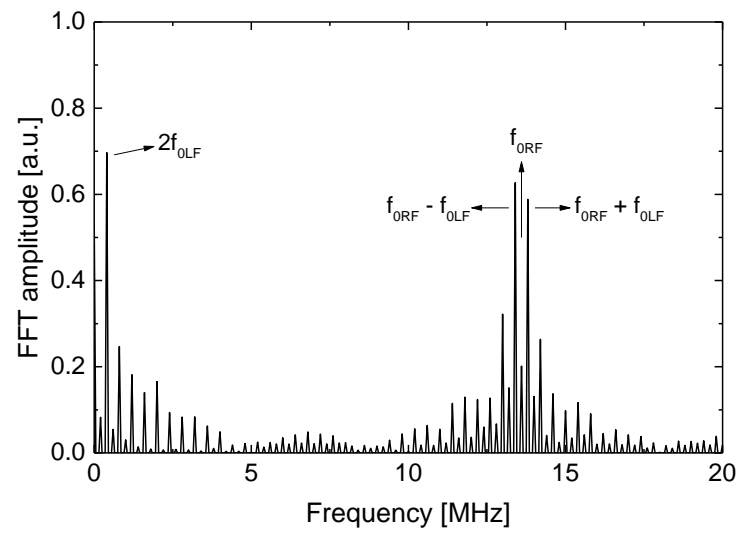

Figure 7. Fast Fourier transform of the phase-resolved emission intensity of the DF discharge in figure 4(d).

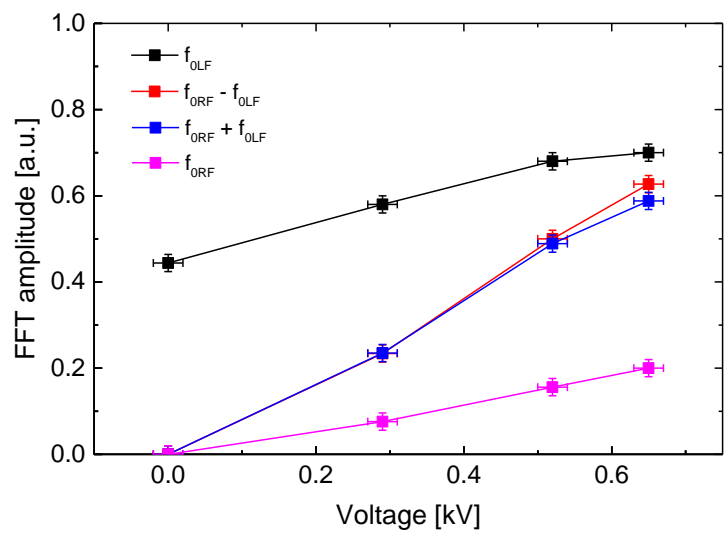

Figure 8. Variations of the FFT amplitude of $f_{\mathrm{LF}}, f_{\mathrm{RF}}$ and $f_{\mathrm{RF}} \pm f_{\mathrm{LF}}$ signals as a function of $U_{\mathrm{RF}}$. 
In summary, atmospheric-pressure dielectric barrier discharges using a $200 \mathrm{kHz} / 13.56 \mathrm{MHz}$ dualfrequency (DF) excitation was studied. It is shown that a combination of LF and RF leads to a discharge strongly modulated by both frequencies, providing a higher input power and an improved discharge uniformity. In the continuously running discharge, the ignition starts when the momentary gas voltage reaches the breakdown value $(\sim 0.7 \mathrm{kV}$ in this study). Due to the periodic oscillation of the RF electric field, the discharge is temporally modulated with an enhancement and a suppression during each RF cycle. As a result, the gas ionization is slowed down, making RF modulation an effective approach to improve the uniformity of the discharge at an increased power input.

\section{Acknowledgements}

This work was supported by the Industrial Partnership Programme i31 (APFF) that is carried out under an agreement between FUJIFILM Manufacturing Europe B.V. and FOM, which is part of the Netherlands Organization for Scientific Research (NWO).

\section{References}

[1] Kogelschatz U, Eliasson B and Egli W 1997 Dielectric-Barrier Discharges . Principle and Applications J. Phys. IV Fr. 7 47-66

[2] Kogelschatz U 2003 Dielectric-barrier discharges: Their History, Discharge Physics, and Industrial Applications Plasma Chem. Plasma Process. 23 1-46

[3] Fridman A 2008 Plasma Chemistry (New York: Cambrigde University Press)

[4] Wagner H-E, Brandenburg R, Kozlov K V, Sonnenfeld A, Michel P and Behnke J F 2003 The barrier discharge: basic properties and applications to surface treatment Vacuum 71 417-36

[5] Massines F, Sarra-Bournet C, Fanelli F, Naudé N and Gherardi N 2012 Atmospheric pressure low temperature direct plasma technology: Status and challenges for thin film deposition Plasma Process. Polym. 9 1041-73

[6] Bruggeman P and Brandenburg R 2013 Atmospheric pressure discharge filaments and microplasmas: physics, chemistry and diagnostics J. Phys. D. Appl. Phys. 46464001

[7] Starostin S A, Premkumar P A, Creatore M, De Vries H, Paffen R M J and Van De Sanden M C M 2010 High current diffuse dielectric barrier discharge in atmospheric pressure air for the deposition of thin silica-like films Appl. Phys. Lett. 9661502

[8] Elam F M, Starostin S A, Meshkova A S, van der Velden-Schuermans B C A M, Bouwstra J B, van de Sanden M C M and de Vries H W 2017 Atmospheric pressure roll-to-roll plasma enhanced CVD of high quality silica-like bilayer encapsulation films Plasma Process. Polym. 141600143

[9] Massines F and Rabehi A 1998 Experimental and theoretical study of a glow discharge at atmospheric pressure controlled by dielectric barrier J. Appl. Phys. 83 2950-7

[10] Bazinette R, Paillol J, Lelièvre J-F and Massines F 2016 Atmospheric Pressure Radio-Frequency DBD Deposition of Dense Silicon Dioxide Thin Film Plasma Process. Polym. 13 1015-24

[11] Fanelli F, d'Agostino R and Fracassi F 2007 Atmospheric pressure PE-CVD of fluorocarbon thin films by means of glow dielectric barrier discharges Plasma Process. Polym. 4 797-805

[12] Fanelli F, Renzo G Di, Fracassi F and D'Agostino R 2009 Recent advances in the atmospheric pressure PE-CVD of fluorocarbon films: Influence of air and water vapour impurities Plasma Process. Polym. 6 503-7

[13] Fanelli F, D'Agostino R and Fracassi F 2011 Effect of gas impurities on the operation of dielectric barrier discharges fed with He, Ar, and Ar-C3F6 Plasma Process. Polym. 8 557-67

[14] Golubovskii Y B, Maiorov V A and Behnke J F 2002 Influence of interaction between charged particles and dielectric surface over a homogeneous barrier discharge in nitrogen J. Phys. D. Appl. Phys. 35 751-61

[15] Massines F, Gherardi N, Naudé N and Ségur P 2005 Glow and Townsend dielectric barrier discharge in various atmosphere Plasma Phys. Control. Fusion 47 B577-88

[16] Bosan D A, Jovanovic T V and Krmpotic D M 1997 The role of neutral metastable molecules in the breakdown probability and glow discharge in nitrogen J. Phys. D. Appl. Phys. 30 3096-8 
[17] Massines F, Gherardi N, Naudé N and Ségur P 2009 Recent advances in the understanding of homogeneous dielectric barrier discharges Eur. Phys. J. Appl. Phys. 4722805

[18] Khamphan C, Segur P, Massines F, Bordage M, N. Gherardi and Cesses Y 2003 Secondary electron emission by nitrogen metastable states in atmospheric-pressure glow discharge 16th Int. Symp. plasma Chem. 589

[19] Aldea E, Peeters P, De Vries H and Van De Sanden M C M 2005 Atmospheric glow stabilization. Do we need pre-ionization? Surf. Coatings Technol. 200 46-50

[20] Gherardi N, Gouda G, Gat E, Ricard A and Massines F 2000 Transition from glow silent discharge to micro-discharges in nitrogen gas Plasma Sources Sci. Technol. 9 340-6

[21] Bazinette R, Subileau R, Paillol J and Massines F 2014 Identification of the different diffuse dielectric barrier discharges obtained between $50 \mathrm{kHz}$ to $9 \mathrm{MHz}$ in $\mathrm{Ar} / \mathrm{NH} 3$ at atmospheric pressure Plasma Sources Sci. Technol. 2335008

[22] Starostin S A, Premkumar P A, Creatore M, van Veldhuizen E M, de Vries H, Paffen R M J and van de Sanden M C M 2009 On the formation mechanisms of the diffuse atmospheric pressure dielectric barrier discharge in CVD processes of thin silica-like films Plasma Sources Sci. Technol. 1845021 